\title{
Energy Landscapes and Dynamics of Glycine on $\mathrm{Cu}(110)$
}

\author{
Marco Sacchi*, David J. Wales ${ }^{\dagger}$ and Stephen J. Jenkins ${ }^{\dagger}$ \\ * Department of Chemistry, University of Surrey, Guildford, GU2 7 XH United Kingdom \\ $\uparrow$ Department of Chemistry, University of Cambridge, Lensfield Road, Cambridge, CB2 1EW, \\ $U K$
}

\begin{abstract}
Amino acids adsorbed over single crystal metal surfaces have emerged as prototypical systems for exploring the properties that govern the development of long-range chirality in selfassembled monolayers (SAM) and supramolecular 2D networks. In this study, we characterise the self-assembly mechanism for glycine on the $\mathrm{Cu}(110)$ surface. This process occurs on a time scale that is too fast for most atomically resolved microscopic techniques, so the mechanism we propose here provides new insight for an important unexplored surface phenomenon.
\end{abstract}

Keywords: self-assembly, amino acids, DFT, glycine, $\mathrm{Cu}(110)$, surface diffusion

\section{Introduction}

Spontaneous self-organization of chiral organic molecules, and in particular of amino acids, on single crystal metal surfaces is of fundamental importance for understanding the mechanisms and phenomena that govern the growth of chiral self-assembled monolayers (SAM) and supramolecular 2D organic networks. ${ }^{1-4}$ Furthermore, adsorption of amino acids on metal surfaces is relevant to nanotechnology and catalysis. ${ }^{5}$ For instance, the hydrogenation of methyl acetoacetate on nickel is an important example of an industrial process that employs amino acids as chiral modifiers for promoting enantioselectivity in this catalytic reaction. ${ }^{6,7}$ 
Adsorption of amino acids on copper surfaces has been intensively studied over the last two decades, both experimentally and theoretically. Copper surfaces are often chosen as substrate because amino acids are stable onto copper up to about $500 \mathrm{~K},{ }^{8}$ while transition metal substrates such as nickel are known to catalyse dissociation even at moderate temperature. ${ }^{9}$ Glycine adsorbed on $\mathrm{Cu}(110)$ is one of the amino acid/ metal systems that has been more thoroughly investigated by diffraction techniques $\left(\mathrm{LEED}^{10,11}, \mathrm{PhD}^{12}\right)$, microscopy $\left(\mathrm{STM}^{11,13}\right)$, IR and X-ray spectroscopy $\left(\right.$ RAIRS $^{11,14}$, NEXAFS $^{10}, \mathrm{XPS}^{8,10}$ ) as well as density functional theory (DFT). ${ }^{15-17}$ More recently, other low-index copper surfaces such as $\mathrm{Cu}(311)$ have been considered for studying glycine selfassembly without footprint chirality. ${ }^{18,19}$

Glycine undergoes facile dehydrogenation upon adsorption on copper surfaces, and is generally present on the surface in its anionic configuration (glycinate), in which the most stable structure is a three-point binding configuration (often represented as $\mu_{3}$ ). Although glycine is not intrinsically chiral, when adsorbed on the $\mathrm{Cu}(110)$ surface it produces the same chiral footprint typical of alanine ${ }^{11,20,21}$ and proline ${ }^{2,22-24}$ on this surface, so glycine can be used as a model to understand the assembly of more complex amino acids on $\mathrm{Cu}(110) \cdot{ }^{2,8,25}$ Even though the bonding, structure and long range arrangement of glycine on $\mathrm{Cu}(110)$ have been investigated by several groups using a wide range of experimental ${ }^{8,13,14,26}$ and computational tools $15,17,27,28$, the energy barriers between adsorbed conformers and the dynamics of glycine surface diffusion are still largely unresolved. In this work we have investigated, using DFT calculations, the energy landscapes of glycine conformers with identical footprint chirality and possible reaction pathways that convert the footprint chirality.

\section{Methods}


The calculations were performed with CASTEP ${ }^{29}$, a plane wave DFT code. The adsorbate has been modelled as a single glycinate adsorbed on a $\mathrm{Cu}(110)-(3 \times 2)$ cell. The surface was modelled by a five layer slab of $(3 \times 2)$ periodicity, with the top two layers allowed to relax during the geometry optimizations and transition state calculations. The Brillouin zone was sampled by a $(4 \times 4 \times 1)$ Monkhorst Pack ${ }^{30}$ k-point grid. As in previous works ${ }^{27}$, we used the Perdew-BurkeErnzerhof $\left(\mathrm{PBE}^{31}\right)$ exchange correlation functional and Vanderbilt ultrasoft pseudopotentials ${ }^{32}$ with a kinetic energy cut-off of $340 \mathrm{eV}$. We performed both single-ended and double-ended transition state search calculations using a combination of CASTEP linear synchronous transit (LST)/ quadratic synchronous transit (QST) method $^{33}$ and hybrid eigenvector-following approach as implemented in the OPTIM program. ${ }^{34-36}$

\section{Results and discussion}

In this work we focus on glycine adsorbed (as glycinate) in a $\mu_{3}$ footprint, with both oxygen and nitrogen atoms bound to the surface on close packed [110] rows, because this is the most stable adsorbed configuration obtained, according to the results of Barlow and coauthors ${ }^{4,14}$, by dosing glycine above $408 \mathrm{~K}$ or by depositing at room temperature followed by annealing at moderate ( 420 K) temperature. ${ }^{4}$ According to the reported RAIRS results, glycine adopts a two point binding arrangement $\left(\mu_{2}\right)$ at low temperature and low coverage, but converts readily to an overall more stable $\mu_{3}$ footprint with increasing coverage and surface temperature. ${ }^{4,}{ }^{14}$ Although it is well understood that glycine (as alanine) adopts a $\mu_{3}$ footprint and a heterochiral (in terms of surface chirality) arrangement ${ }^{15,25}$ in the $(3 \times 2)$ phase, there is relatively little information regarding the energy difference between the $\mu_{3}$ conformers (having the same footprint, but different C-C-N backbone torsional angles) and about how they interconvert. In this work we have initially 
compared the energetics of three $\mu_{3}$ glycine conformers (Figure 1) and we have performed transition state calculations to estimate the energy barriers for conversion between them.

The major configuration changes between the conformers involves the C-C-N backbone orientation and the positions of the oxygen atoms on the surface, while the overall footprint chirality is conserved. The most stable conformer (gly 3) has the C-C-N backbone tilted in the opposite direction of the right angle in the $\mu_{3}$ triangle, and it is about $0.15 \mathrm{eV}$ more stable than conformer gly 1 (with the backbone tilted in the same direction of the right footprint triangle) and $0.14 \mathrm{eV}$ more stable than conformer 2 (with a C-C-N backbone plane essentially perpendicular to the surface. gly 3 is characterized by marked tilt of the $\mathrm{C}-\mathrm{N}$ bond towards one of the oxygen atoms. The stronger hydrogen bonding between one of the $\mathrm{N}-\mathrm{H}$ bonds and the adsorbed oxygen determines the overall greater stability of this conformer (the $\mathrm{H}-\mathrm{O}$ distance $d(\mathrm{O}-\mathrm{H})$ is $2.43 \AA$ ) with respect to gly $1(d(\mathrm{O}-\mathrm{H})=2.73 \AA)$ and gly $2(d(\mathrm{O}-\mathrm{H})=2.51 \AA)$ since other structural parameters such as the lengths of the C-C, C-N bonds and the angle of C-C-N backbone are very similar among the three conformers. An extremely small activation energy $\left(E_{a}=8 \mathrm{meV}\right)$ is sufficient in order to convert gly 1 to gly 2 (the transition state structure is reported in Figure 1), therefore it is likely that, even at low surface temperatures, one would be able to observe spontaneous transitions between these two structures. We have not been able to obtain a converged TS structure between gly 2 and gly 3 , probably because the barrier height for this structural modification is comparable with the numerical errors of our computational methods $(\sim 5 \mathrm{meV})$. Since the binding configuration of gly on $\mathrm{Cu}(110)$ is substantially identical to that of alanine (ala) $\mu_{3}$, the energy barriers we calculated for gly suggest a similarly flat energy landscape for ala on this surface. 


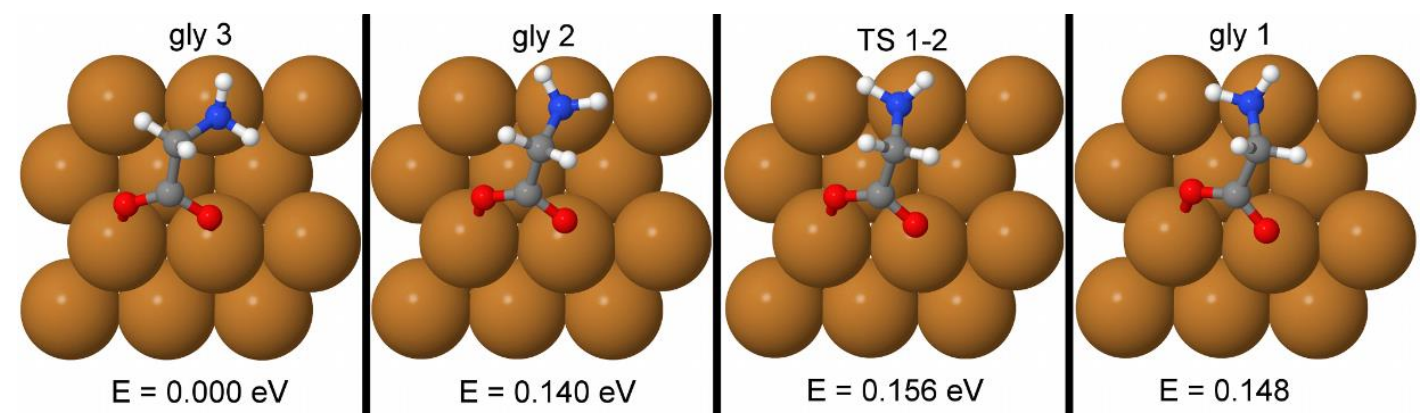

Figure 1. gly 3 , gly 2 and gly 1 are three conformers of glycine adsorbed on $\mathrm{Cu}(110)$ in a $\mu_{3}$ binding configuration. TS $1-2$ is the transition state connecting gly 1 and gly 2 . The barrier height is only $8 \mathrm{meV}$. Using the same hybrid eigenvector-following method ${ }^{34,36}$ we have not been able to find a converged TS connecting gly 2 and gly 3 , and it is likely that the energy barrier between these two conformers is lower than the numerical error in the DFT calculations (about 5 $\mathrm{meV})$. 
The mechanism by which gly diffuses along the $\mathrm{Cu}(110)$ steps is unknown. Experimentally, it is necessary to anneal the surface at about $420 \mathrm{~K}$ to produce an ordered $(3 \times 2)$ adlayer, ${ }^{4,14}$ so we expect that gly diffusion is a thermally activated process with a substantial energy barrier. We have investigated the dynamics of gly diffusion in the direction parallel to the $\mathrm{Cu}(110)$ steps by assuming that the molecule changes its footprint chirality after each step (see Figure 2) and that either the amino or the carboxylate group remains bonded to the same site while the other group changes its binding configuration. This is a reasonable assumption because elementary thermodynamics suggests that a molecule would break the minimum number of bonds with the surface during a diffusion step. Both STM and RAIRS agree that the adsorbate is never bound to the surface via a single $\mathrm{Cu}-\mathrm{O}$ or $\mathrm{Cu}-\mathrm{N}$ bond, therefore, although not a priori impossible, a step that includes a TS with a single molecule-to-surface bond would probably be energetically unfavourable. 

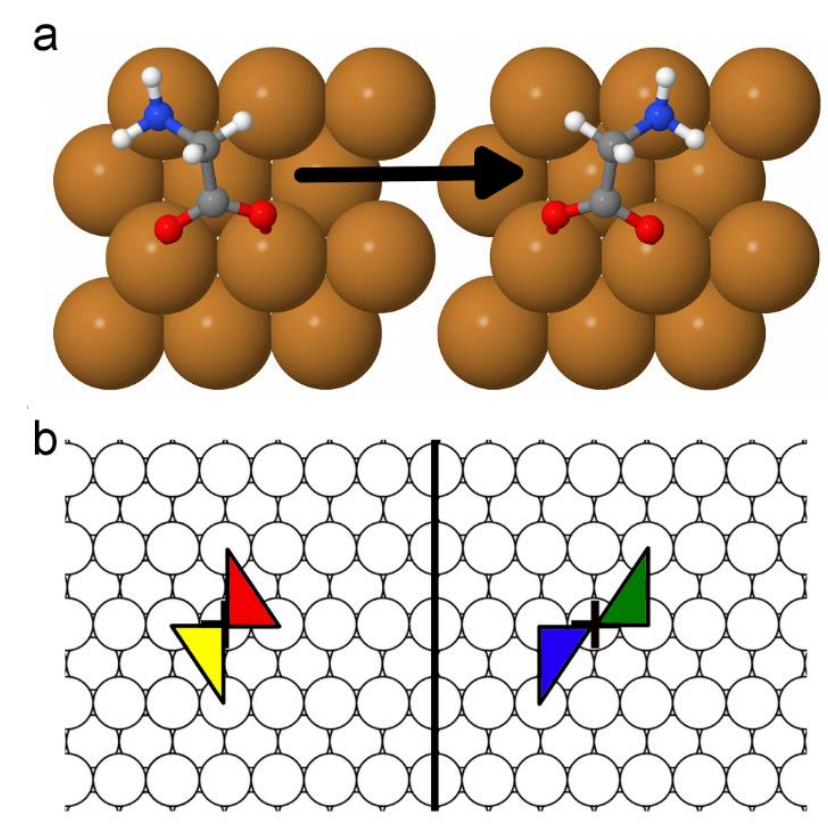

Figure 2. Isolated glycine is non chiral, but when adsorbed on $\mathrm{Cu}(110)$ as glycinate (a) it can populate two energetically equivalent orientations (left and right handed triangular footprint) that create the so called "footprint" chirality. We have investigated the energy barrier between these two enantiomers for three different diffusion pathways. A schematic representation of the footprint chirality transition is depicted in panel (b) from red (initial "left" chirality) to green (final "right" chirality). For completeness, the two other footprint orientations, yellow and blue triangles (having the same chirality of red and green respectively) are also shown in panel (b). 
To start with, four diffusion pathways for diffusion along the close packed rows were considered (see Figure 3). In the first diffusion mechanism the $\mathrm{NH}_{2}$ group of gly hops from an atop site to an adjacent atop site passing through a transition state (TS 1, Figure 3) in which the nitrogen atom lies at a bridge site. The second mechanism involves the synchronous shifting along the [110] direction of the two $\mathrm{O}$ atoms. At the transition state (TS 2, Figure 3), the molecule is in a pseudo- $\mu_{3}$ configuration, with an $\mathrm{O}$ atom bonded on a bridge site and the other $\mathrm{O}$ in an off-atop position at a slightly longer distance from the surface with respect to the first oxygen. The third pathway can be described as a $180^{\circ}$ rotation of the OCO group around one of the bonded $\mathrm{O}$ atoms. At the TS (TS 3, Figure 3) the molecule is in a two-points binding configuration $\left(\mu_{2}\right)$ with the $\mathrm{N}$ atom bonded on an atop site and an $\mathrm{O}$ atom on a long-bridge site between two steps. $\mathrm{The}^{\mathrm{N}} \mathrm{NH}_{2}$ hopping mechanism has the lowest activation energy $(0.358 \mathrm{eV})$, but the OCO shifting mechanism has a very similar energy barrier (only about $15 \mathrm{meV}$ higher), so it is likely that, at the surface temperatures generally used in experiments, gly surface diffusion could proceed via both pathways. The mechanism that involves the OCO rotation has an energy barrier $0.18 \mathrm{eV}$ higher than the other two other diffusion pathways, so it is less probable that gly diffusion will involve the breaking of single $\mathrm{Cu}-\mathrm{O}$ bond with the molecule passing through a $\mu_{2}$ TS configuration. The last diffusion pathway (pathway 4, in Figure 3) explored is similar to pathway 3 described above, with a $\mu_{2}$ transition state involving the binding of nitrogen on an atop site and the binding of only a single oxygen of the carboxylic group on a bridge site. This pathway differs from pathway 3 by the sense of rotation of the OCO group: in pathway 3 the oxygen is initially on the left side of the reactant, breaking its bond with the surface and forming another bond on the right of the molecule, while in pathway 4 the oxygen is initially on the right side of glycine and rotates in the opposite direction. Each path from 1 to 4 involves a change in footprint chirality, and each path involves 
either a change in the nitrogen or oxygen position on the surface. A complete diffusion step, which conserves the initial chirality and involves a complete motion of the molecule (both $\mathrm{N}$ and OCO changing position) along the rows, can therefore be described by a combination of path $1+$ path ( 2 or 3 or 4). Since path 2 is the most energetically favourable mechanism for shifting the position of the OCO group along the rows, the overall activation barrier for diffusion in this direction will be close to $0.372 \mathrm{eV}$.

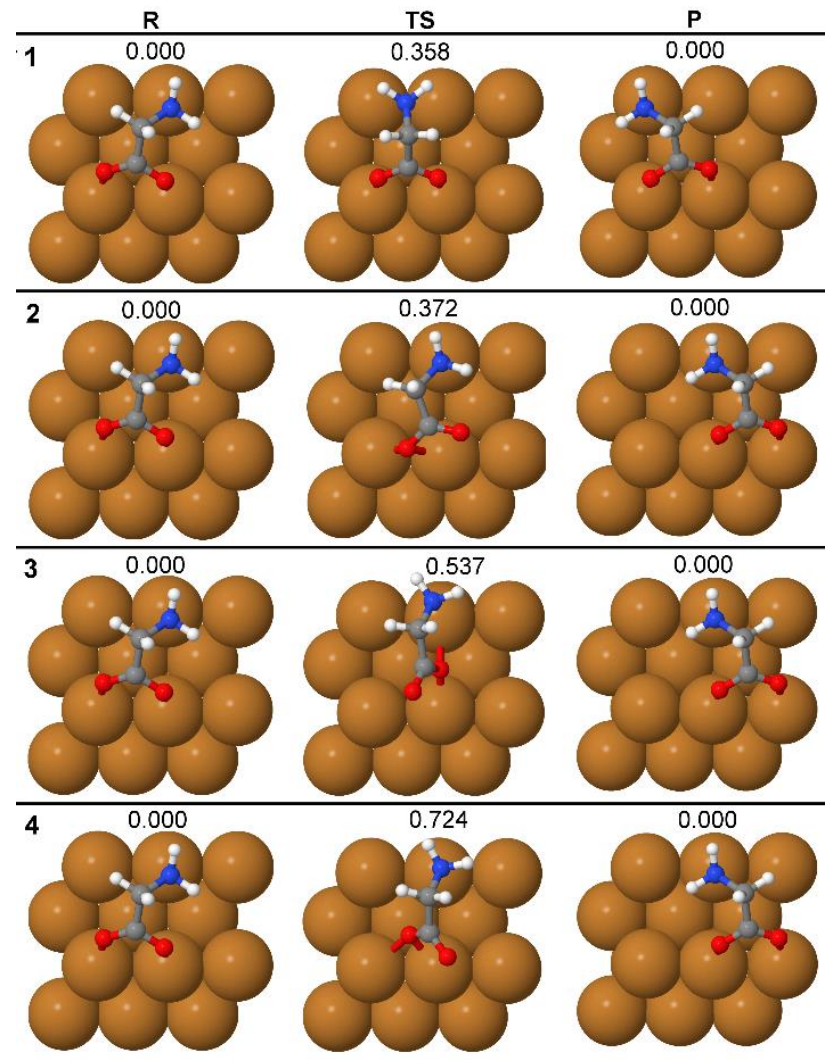

Figure 3. Paths 1 to 4 illustrate the transition state structures and initial and final energies (eV) (the energy values are relative to the reactant) for glycine conversion from a left to right footprint chirality through four different rearrangement mechanisms. The panels are reactant (R), transition state (TS), and product (P), from left to right. 
We now consider the motion of glycine across the close packed rows, in the [001] and equivalent directions. This is an essential part of the surface diffusion process that allows the molecules to form hydrogen bonds between the carboxylate and the amino groups in molecules occupying alternating rows. In Figure 4, path 5 describes glycine changing from a $\mu_{3}$ configuration to an intermediate standing configuration by breaking the $\mathrm{Cu}-\mathrm{N}$ bond on one row. The associated barrier is $0.384 \mathrm{eV}$ and the transition state configuration is very close in geometry and energy to the stable $\mu_{2}$ intermediate $(\mathrm{P})$, in which the molecule binds with the surface through the oxygen atoms of the OCO group only and there is the formation of an internal $\mathrm{N}-\mathrm{H}-\mathrm{O}$ hydrogen bond that helps reducing the energy demands for this step. From this $\mu_{2}$ intermediate configuration $(0.332 \mathrm{eV})$ the $\mathrm{NH}_{2}$ group can adsorb back on an adjacent row. This diffusion step is summarized, with the four possible initial and final configurations (depending on the initial footprint, left or right oriented) in Figure 5 a-d. This motion allows the molecule to change both footprint chirality and orientation, by binding two different close-packed rows at the end of the $\mathrm{NH}_{2}$ "jump", but, since the carboxylic groups remain bound to the same site, a repetition of multiple jumps will not allow the molecule to diffuse across the rows. For an overall diffusion in a direction perpendicular to the close packed rows to occur, both binding groups need to move. This process is shown in Figure 4 path 6, in which the glycinate, initially in the $\mu_{3}$ minimum energy configuration, undergoes a concerted translation and rotational motion that allows it to shift its centre of mass towards the close packed row that is bound to the amino group. The barrier for this rearrangement is only about $0.4 \mathrm{eV}$ and the molecule finishes in a higher energy $\mu_{3}$ configuration, in which the OCO is on a long-bridge site, binding with two adjacent close-packed rows while the $\mathrm{NH}_{2}$ group is still bound to the same copper atom, but moves from an atop site to an off-top site. This is the first step of the mechanism that allows the molecule to move across the close packed rows. From this high-energy $\mu_{3}$ 
configuration the $\mathrm{NH}_{2}$ group can hop across the ridge site and bind onto the opposite row (Figure 4 path 7), ending up in a $\mu_{3}$ long-bridge configuration which is the mirror-image of the initial structure. The activation energy for this step is $0.610 \mathrm{eV}$, which is substantially higher than the two previous steps. Clearly, from this intermediate position the molecule can shift back to a global minimum configuration by repeating step 6 , but in the opposite direction. Figure 4 path 8 shows an alternative mechanism allowing the molecule to switch the binding site of the amino group from a long-bridge $\mu_{3}$ configuration. In this diffusion path, the $\mu_{3}$ long-bridge configuration changes from a long-bridge $\mu_{3}$ to a vertical $\mu_{2}$ geometry through a transition state having slightly lower energy barrier ( 0.582 instead of $0.610 \mathrm{eV})$. From this high-energy "standing" position the $\mathrm{NH}_{2}$ can easily reattach to the surface on either side of the molecule, allowing the molecule to move across the rows and/or switch footprint chirality (Figure 5, i to n). The overall motion across the close packed rows is accomplished by a repetition of single steps 5 to 7 (or 8) and then 7 (or 8) to 1 in succession (Figure 5, i and $\mathrm{j}$ ).

To summarize, in Figure 5, one can see how each step can produce either a chiral change (from green to red triangle and from yellow to blue triangle), an orientation change (from green to blue triangle and from red to yellow triangle) or both (from green to yellow triangle and from red to blue triangle). A simple chiral change or orientation change, especially when it involves the breaking of a single $\mathrm{N}-\mathrm{Cu}$ bond (Figure 5, steps a to f), happens much faster than a step involving the hopping of the OCO group from one row to an adjacent row (Figure 5, steps i to 1). Furthermore, while a change in chirality or orientation may involve a single hopping mechanism that starts from glycine in a $\mu_{3}$ configuration and ends with the adsorbate on an energetically equivalent $\mu_{3}$ configuration, when the OCO group jumps to another row, intermediate vertical and horizontal configurations can be present. While these energetically unfavourable configurations will have a 
relatively short life time at low coverage or high surface temperature, it is possible that at lower temperature or at coverage close to saturation these $\mu_{2}$ species might be seen in IR spectra, as suggested by Barlow et al. ${ }^{4,14}$

The combination of motion along and across the close packed rows and the ability to switch footprint chirality (the calculated barrier heights are compatible with surface phenomena easily happening at room temperature) allow the adsorbed monomers to join together in islands formed by interlinked, hydrogen bonded, heterochiral ${ }^{25}$ domains (Figure 6). The DFT results relative to the $(3 \times 2)$ heterochiral phase were reported in our previous work on this system. ${ }^{27}$ We reproduce the structure of the $(3 \times 2)$ phase here for illustrative purposes, to show how the triangles used in Figure 2 and Figure 5 to represent the adsorbed glycine $\left(\mu_{3}\right)$ fit in a heterochiral arrangement at saturation coverage.

A direct comparison between our computational results and the available experimental studies is not straightforward since the great majority of the experimental work reported in literature involves a high coverage of glycine (close to $1 \mathrm{ML}$ ) and poor time-resolution (on the order of several minutes for STM, RAIRS and XPS studies). As previously observed, the relatively high annealing temperature, around $420 \mathrm{~K}$ (compared to a desorption temperature, of about $500 \mathrm{~K}$ ), needed for obtaining a $(3 \times 2)$ ordered phase on $\mathrm{Cu}(110)$ is indicative of an activated mass-transport process with a consistent barrier height. Since the calculated a binding energy $\left(E_{a}\right)$ of glycine at low coverage is $0.86 \mathrm{eV},{ }^{27}$ one would expect an activation energy for diffusion $\left(\mathrm{E}_{\mathrm{d}}\right)$ of about $0.26 \mathrm{eV}$ (according to the very approximate semiempirical formula $E_{d}=\alpha E_{a}$, with $\alpha=0.3$ ). The significant difference in activation energy (about $0.2 \mathrm{eV}$ ) between diffusion along the close-packed rows (Figure 3) and diffusion across the close-packed rows (Figure 4) suggests that the growth mechanism of glycine (and possibly alanine, given the close similarity between the two molecules) 
would be essentially anisotropic. Although there is no evidence in the literature of anisotropic mass transport of glycine and alanine on $\mathrm{Cu}(110)$, there is clear evidence that serine growth on the same surface proceeds by preferential diffusion along specific directions. ${ }^{37}$ Even accounting for the different diffusion step $(a)$ in the two directions ( $a=2.56 \AA$ along the rows and $a=3.62 \AA$ across the rows), the diffusion coefficient $\mathrm{D}=v a^{2} / z$ (where $v$ is the hopping frequency and $z$ is the number of nearest neighbours) for glycine diffusion along the rows would be at least a factor of 100 higher than for motion across the rows, at moderate temperatures $(350-450 \mathrm{~K})$.

From the RAIRS spectrum at low coverage, Barlow et al postulated the presence of an upright orientation of glycine on $\mathrm{Cu}(110)$, with the adsorbate bonded to the surface by the OCO group only. ${ }^{14}$ We note that the observed vertical orientation of glycine on $\mathrm{Cu}(110)$ is probably not the result of the presence of a transient glycine species in thermal equilibrium. Our calculations for diffusion paths across the close-packed rows (see Figure 4, panel 5 and 8) predict that the vertical and $\mu_{2}$ species are about 0.37 and $0.38 \mathrm{eV}$ higher in energy than the global minimum, therefore the Boltzmann population of these species would about $10^{5}$ times lower than that of the global minimum at $450 \mathrm{~K}$. Since the IR features of the vertical glycine configuration were not observed when the coverage increased and the surface was annealed at $420 \mathrm{~K}$, it is likely that the vertical and $\mu_{2}$ configurations are only metastable species at low coverage (somehow stabilized by defects or clustering) and not real global minima, in agreement with our results. 


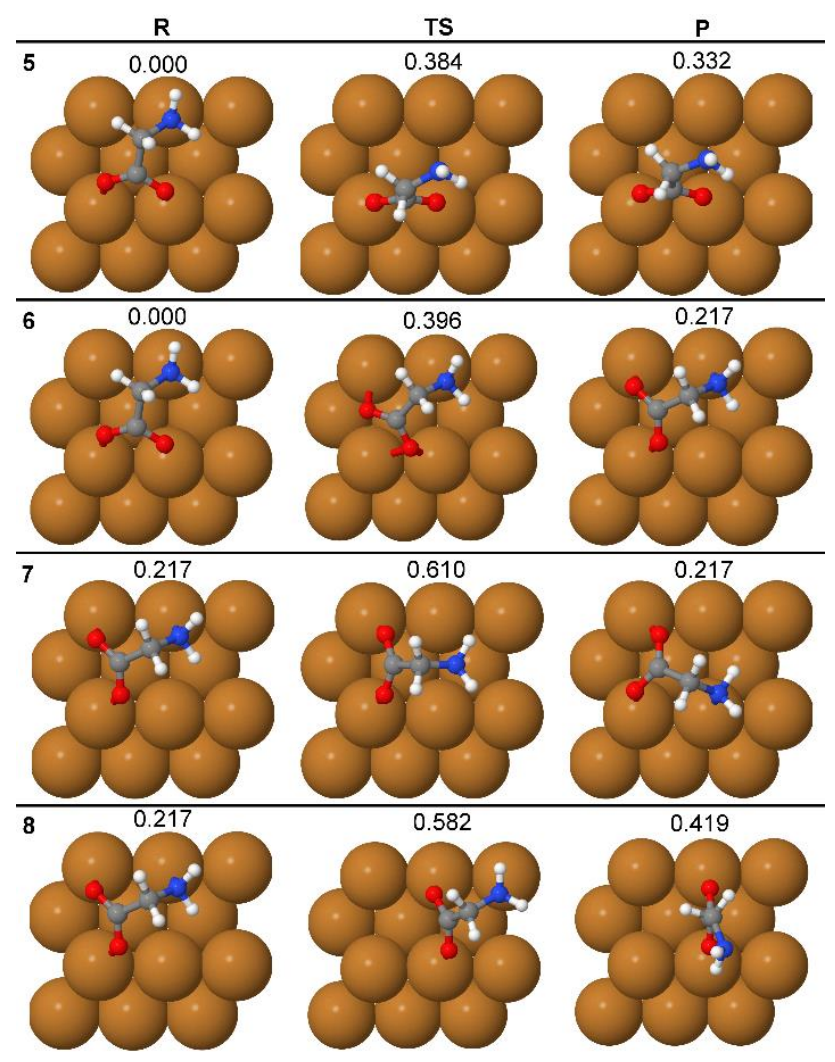

Figure 4 Path 5 to 8 illustrate the transition state structures and initial and final energies (eV) (the energy values are relative to the reactant) for glycine moving across the close packed rows. Only the first part of the diffusion path is shown. The panels are reactant (R), transition state (TS), and product $(\mathrm{P})$, from left to right. 

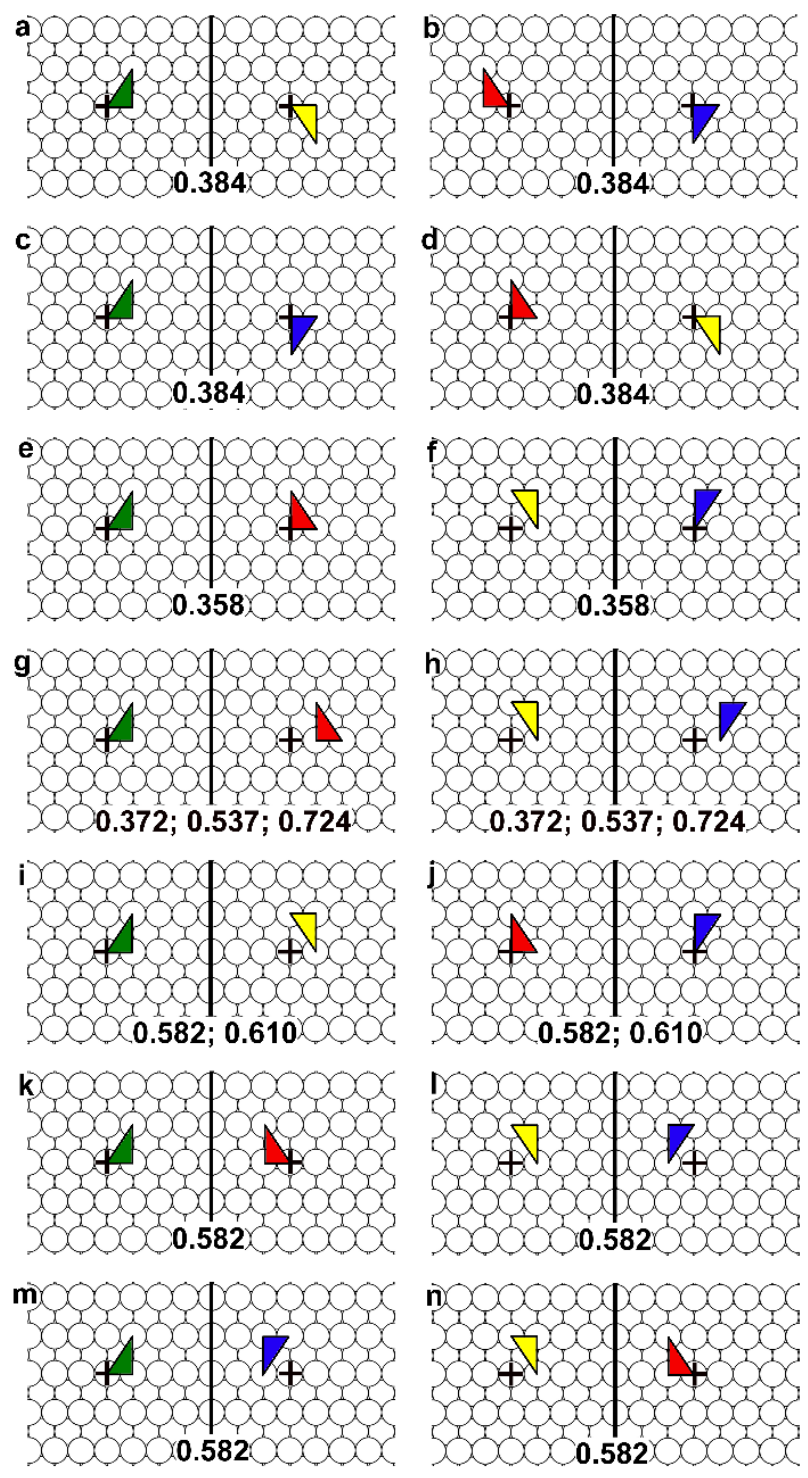

Figure 5 Summary of the $\mu_{3}$ configuration transitions, only the initial and final configurations are shown. The four possible, energetically equivalent, $\mu_{3}$ configurations are represented by triangles. Green and blue, and red and yellow triangles have same footprint chirality, but different surface orientation. The chiral enantiomers of green and blue are red and yellow, respectively. The activation barrier $(\mathrm{eV})$ for the single step, or the highest barrier for the multistep mechanisms (motion across the rows), are also reported. For steps g-1, multiple paths are available, so we report the barriers for each of them. 

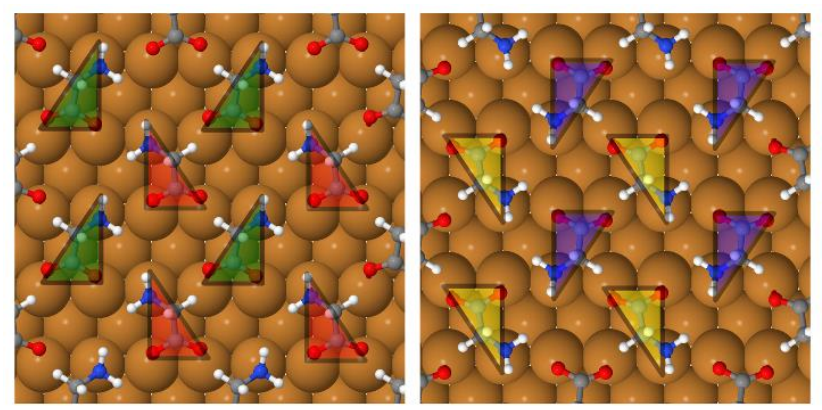

Figure 6 The result of diffusion and self-assembly is island and terrace formation for glycine bound by hydrogen bonds in $(3 \times 2)$ heterochiral domains. The DFT results relative to this configuration can be found in our previous work ${ }^{27}$. In this figure, the green-red island (left panel) and the yellow-blue island (right panel) are identical in terms of overall (racemic) chirality (and energy), but are oriented in opposite directions with respect to the substrate. 


\section{Conclusions}

The DFT modelling for diffusion of glycine on copper offers unique insights into the complex dynamical process of amino acid self-assembly on metal surfaces. Glycine diffusion along and across the close-packed rows of the $\mathrm{Cu}(110)$ surface proceeds through a step-by-step "walking" mechanism in which the molecule is able to shift, rotate and change orientation by breaking only a single surface bond at the time. Depending on the path, the amino acid can retain or invert the original chiral footprint, therefore allowing the monomer to form long-range heterochiral terraces on the surface. The activation energies calculated confirm that that self-assembly of glycine on low-index copper surfaces is indeed an activated process, which is facile in the temperature range (typically from $300 \mathrm{~K}$ to $420 \mathrm{~K}$ ) generally employed in experiments. We suggest that the walking mechanism predicted in this study might play a key role in the formation of similar self-assembled systems relevant for nanotechnology and surface chemistry, such as thiolates ${ }^{38}$, amines ${ }^{39}$ or chiral organic acids ${ }^{40}$ on metal surfaces.

\section{Acknowledgements}

MS would like to thank the Royal Society for his University Research Fellowship.

\section{References}

1. A. G. Mark, M. Forster and R. Raval, ChemPhysChem, 2011, 12, 1474-1480.

2. M. Forster, M. Dyer, M. Persson and R. Raval, Top. Catal., 2011, 54, 13-19.

3. S. M. Barlow and R. Raval, Curr. Opin. Colloid Interface Sci., 2008, 13, 65-73.

4. S. M. Barlow and R. Raval, Surf. Sci. Rep., 2003, 50, 201-341.

5. Y. Zhao, B. Ashcroft, P. Zhang, H. Liu, S. Sen, W. Song, J. Im, B. Gyarfas, S. Manna, S. Biswas, C. Borges and S. Lindsay, Nat Nano, 2014, 9, 466-473.

6. C. J. Baddeley, Top. Catal., 2003, 25, 17-28.

7. A. Wolfson, S. Geresh, M. V. Landau and M. Herskowitz, Applied Catalysis A: General, 2001, 208, 91-98. 
8. A. Shavorskiy, F. Aksoy, M. E. Grass, Z. Liu, H. Bluhm and G. Held, J. Am. Chem. Soc., 2011, 133, 6659-6667.

9. R. E. J. Nicklin, A. Cornish, A. Shavorskiy, S. Baldanza, K. Schulte, Z. Liu, R. A. Bennett and G. Held, The Journal of Physical Chemistry C, 2015, 119, 26566-26574.

10. J. Hasselström, O. Karis, M. Weinelt, N. Wassdahl, A. Nilsson, M. Nyberg, L. G. M. Pettersson, M. G. Samant and J. Stöhr, Surf. Sci., 1998, 407, 221-236.

11. S. Haq, A. Massey, N. Moslemzadeh, A. Robin, S. M. Barlow and R. Raval, Langmuir, 2007, 23, 10694-10700.

12. N. A. Booth, D. P. Woodruff, O. Schaff, T. Gießel, R. Lindsay, P. Baumgärtel and A. M. Bradshaw, Surf. Sci., 1998, 397, 258-269.

13. Q. Chen, D. J. Frankel and N. V. Richardson, Surf. Sci., 2002, 497, 37-46.

14. S. M. Barlow, K. J. Kitching, S. Haq and N. V. Richardson, Surf. Sci., 1998, 401, 322-335.

15. R. B. Rankin and D. S. Sholl, Surf. Sci., 2004, 548, 301-308.

16. S. Blankenburg and W. G. Schmidt, Nanotechnology, 2007, 18, 424030.

17. J. W. Han, J. N. James and D. S. Sholl, The Journal of Chemical Physics, 2011, 135, 034703.

18. D. C. Madden, M. L. Bentley, S. J. Jenkins and S. M. Driver, Surf. Sci., 2014, 629, 81-87.

19. D. C. Madden, I. Temprano, M. Sacchi and S. J. Jenkins, The Journal of Physical Chemistry C, 2015, 119, 13041-13049.

20. J. Seifert, M. Busch, E. Meyer and H. Winter, Physical Review B, 2014, 89, 075404.

21. G. Jones, L. B. Jones, F. Thibault-Starzyk, E. A. Seddon, R. Raval, S. J. Jenkins and G. Held, Surf. Sci., 2006, 600, 1924-1935.

22. M. Forster, M. S. Dyer, M. Persson and R. Raval, Angew. Chem. Int. Ed., 2010, 49, 23442348.

23. M. Forster, M. S. Dyer, M. Persson and R. Raval, J. Am. Chem. Soc., 2009, 131, 1017310181.

24. E. Mateo Marti, S. M. Barlow, S. Haq and R. Raval, Surf. Sci., 2002, 501, 191-202.

25. Z. V. Zheleva, T. Eralp and G. Held, The Journal of Physical Chemistry C, 2011, 116, 618625.

26. V. Efstathiou and D. P. Woodruff, Surf. Sci., 2003, 531, 304-318.

27. M. Sacchi and S. Jenkins, PCCP, 2014, 16, $6101-6107$.

28. S. Monti, C. Li and V. Carravetta, J. Phys. Chem. C, 2013, 117, 5221-5228.

29. S. J. Clark, M. D. Segall, C. J. Pickard, P. J. Hasnip, M. J. Probert, K. Refson and M. C. Payne, Zeitschrift Fur Kristallographie, 2005, 220, 567-570.

30. H. J. Monkhorst and J. D. Pack, Physical Review B, 1976, 13, 5188.

31. J. P. Perdew, K. Burke and M. Ernzerhof, Phys. Rev. Lett., 1996, 77, 3865-3868.

32. D. Vanderbilt, Physical Review B, 1990, 41, 7892.

33. N. Govind, M. Petersen, G. Fitzgerald, D. King-Smith and J. Andzelm, Computational Materials Science, 2003, 28, 250-258.

34. Y. Kumeda, D. J. Wales and L. J. Munro, Chem. Phys. Lett., 2001, 341, 185-194.

35. D. J. Wales, Energy Landscapes, Cambridge University Press, Cambridge, 2003.

36. L. J. Munro and D. J. Wales, Physical Review B, 1999, 59, 3969.

37. T. Eralp, A. Shavorskiy, Z. V. Zheleva, G. Held, N. Kalashnyk, Y. Ning and T. R. Linderoth, Langmuir, 2010, 26, 18841-18851.

38. J. C. Love, L. A. Estroff, J. K. Kriebel, R. G. Nuzzo and G. M. Whitesides, Chem. Rev., 2005, 105, 1103-1170. 
39. A. D. Gordon and F. Zaera, Angew. Chem. Int. Ed., 2013, 52, 3453-3456.

40. V. Demers-Carpentier, A. M. H. Rasmussen, G. Goubert, L. Ferrighi, Y. Dong, J.-C. Lemay, F. Masini, Y. Zeng, B. Hammer and P. H. McBreen, J. Am. Chem. Soc., 2013, 135, 9999-10002. 